\title{
Kalsiyum floroborat sentezi, kinetik ve alev geciktirici özelliklerinin belirlenmesi
}

\author{
Metin Gürü1', Gülden Güngör², Duygu Y. Aydınn ${ }^{3 *}$, Çetin Çakanyıldırım \\ ${ }^{1}$ Gazi Üniversitesi, Mühendislik Fakültesi, Kimya Mühendisliği Bölümü, Ankara, 06570, Türkiye \\ ORCID orcid.org/0000-0002-7335-7583 \\ ${ }^{2}$ TENMAK Bor Araştırma Enstitüsü, Ankara, 06530, Türkiye ORCID orcid.org/0000-0003-1517-7821 \\ ${ }^{3}$ Malatya Turgut Özal Üniversitesi, Mühendislik ve Doğa Bilimleri Fakültesi, Biyomühendislik Bölümü, Malatya, 44210, Türkiye \\ ORCID orcid.org/0000-0003-0557-5279 \\ ${ }^{4}$ Hitit Üniversitesi, Mühendislik Fakültesi, Kimya Mühendisliği Bölümü, Çorum, 19030, Türkiye \\ ORCID orcid.org/0000-0001-7040-1369
}

\section{MAKALE BİLGISi}

Makale Geçmişi:

İlk gönderi 14 Şubat 2021

Kabul 28 Haziran 2021

Online 30 Eylül 2021

Araştırma Makalesi

DOI: $10.30728 /$ boron.880116

Anahtar kelimeler:

Alev geciktirici

Floroborik asit

Kalsiyum floroborat

LOI

\begin{abstract}
ÖZET
$\mathrm{Bu}$ çalışmada özel bor bileşiklerinden olan kalsiyum floroboratın sentez parametreleribelirlenmişve pamuklu kumaştakialevgeciktiriciözelliği incelenmiştir. Ayrıca kinetik çalışmalar yapılarak reaksiyon mertebesi ve aktivasyon enerjisi hesaplanmıştır. Kalsiyum floroborat, reaktant olarak kalsiyum oksit ve floroborik asit kullanılarak yaş yöntemle sentezlenmiştir. İncelenen parametreler; reaktant mol oranı $\left(\mathrm{nCaO} / \mathrm{nHBF}_{4}\right)$, sıcaklık ve reaksiyon süresidir. Karakterizasyon çalışmaları için FT-IR, XRD ve $\mathrm{BF}_{4}$ - iyon seçici elektrot kullanılmıştır. Termal davranışın karakterize edilmesinde termogravimetrik-diferansiyel termal analiz (TG-DTA) kullanılmıştır. Kalsiyum floroborat, $1: 4$ reaktant mol oranı, $90^{\circ} \mathrm{C}$ sıcaklık ve 100 dakika reaksiyon süresinde $\% 97$ verimle sentezlenmiştir. Ayrıca yapılan kinetik çalışmada reaksiyonun birinci mertebeden olduğu belirlenmiş ve reaksiyonun aktivasyon enerjisi $19,14 \mathrm{~kJ} / \mathrm{mol}$ olarak bulunmuştur. Sentezlenen kalsiyum floroboratın alev geciktirici özelliğini tespit etmek için LOI testinden yararlanılmıştır. Testler sonucunda kalsiyum floroboratın çok iyi alev geciktirici özellik gösterdiği gözlemlenmiştir.
\end{abstract}

\section{Calcium fluoroborate synthesis, determination of kinetics and flame retardant properties}

\section{ARTICLE INFO}

Article history:

Received February 14, 2021

Accepted June 28, 2021

Available online September 30, 2021

Research Article

DOI: $10.30728 /$ boron. 880116

\section{Keywords:}

Flame retardant

Calcium fluoroborate

Fluoroboric acid

LOI

\begin{abstract}
In this study, the synthesis parameters of calcium fluoroborate, one of the special boron compounds, were determined and its flame retardant properties in cotton fabric were investigated. In addition, kinetic studies were carried out to calculate the reaction order and activation energy. Calcium fluoroborate was synthesized by wet method using calcium oxide and fluoroboric acid as reactants. The parameters examined were reactant mole ratio $\left(\mathrm{nCaO} / \mathrm{nHBF}_{4}\right)$, temperature and reaction time. FT-IR, XRD and $\mathrm{BF}_{4}$ - ion selective electrode were used for characterization studies. Thermogravimetric-differential thermal analysis (TG-DTA) was used to characterize the thermal behavior. Calcium fluoroborate was synthesized with $97 \%$ yield when molar ratio of reactants, temperature and reaction time are set as $1: 4,90^{\circ} \mathrm{C}$ and 100 minutes, respectively. In addition, kinetic experiments reveal that the reaction order obeys to first order kinetics and the activation energy was found as $19.14 \mathrm{~kJ} / \mathrm{mol}$. LOI tests were used to determine the flame retardant properties of synthesized calcium fluoroborate. Tests proved that the calcium fluoroborate has very good flame retardant properties.
\end{abstract}

\section{Giriş (Introduction)}

Dünyada ticari olarak üretilip değişik alanlarda kullanılan ve nihai ürün olarak sınıflandırılabilen birçok özel bor bileşiği mevcuttur. Bu bileşiklerin her biri farklı amaçlar için değişik sektörlerde kullanılmaktadır. Bu ürünlerden kullanım alanı en yaygın olanları; potasyum borhidrür, sodyum borhidrür, boranlar, susuz bo- 
rik asit (bor oksit), metal borürler, disodyum oktaborat tetrahidrat, çinko borat, bor triklorür, bor karbür, ferrobor, bor fiberleri, metalik (elementel) bor ve bor nitrür olarak sıralanabilir [1]. Özel bor bileşiklerinden birisi olan floroboratlar, floroborik asidin tuzları olup ilk bilimsel çalışmalar Berzelius tarafından yapılmıştır [2]. Bu gruptaki bileşikler, alkali metal floroboratlar, amonyum floroborat ve geçiş element floroboratlarıdır [3]. Metal floroboratlar; floroborik asit ve metal tuzlarından veya borik asit ya da hidroflorik asidin metal tuzları ile reaksiyonundan elde edilir [4]. Ayrıca floroboratlar; metal florür ve elementel borun katı faz reaksiyonu ile elde edilebilmektedirler $[5,6]$.

Floroboratlar endüstrinin çeşitli alanlarında farklı kullanım alanları bulmaktadırlar. Kalsiyum floroborat, nadir toprak elementleriyle birlikte camlarda kullanıldıklarında fiziksel parametreleri iyileştirirler ve devitrifikasyona karşı termal kararılıık sağlarlar [7-9]. Ayrıca lityum iyon pillerinde ve kalsiyum kaplamalarda elektrolit olarak kullanımaktadırlar [10,11]. Metal floboratlardan biri olan çinko floroborat, çeşitli reaksiyonlarda katalizör olarak, tekstil endüstrisinde buruşmazlık apresi reçinelerinde kür kimyasalı olarak kullanılmaktadır [1214]. Lityum floroborat, lityum sülfür pillerinde elektrolit olarak kullanılmaktadır. Lityum floroborat bazlı elektrolit kullanan Li-iyon hücresi neme karşı daha az duyarlıdır ve yüksek sıcaklıkta elektrolitte çok daha iyi performans göstermektedir [15]. Potasyum floroboratın elektrolizi ile yüksek saflıkta elementel bor elde edilebilmektedir [16]. Kalay floroborat, kaplamalarda kullanılmaktadır. Yüksek çözünürlüğü ve iyi elektrik iletkenliği sayesinde, yüksek akım yoğunluğu uygulanabilmektedir [17]. Floroborat banyoları, yüksek akım verimlilikleri olmasının yanında düşük gerilime sahip, katkı maddeleri gerektirmeyen ve safsızlıklara karşı çok az bir hassasiyet sağlayan katmanlar sağlamaktadırlar [18].

Diğer yandan floroboratlar malzemelerin alev direncini artırıllar. Floroboratlar; sentetik liflerde, polimerlerde [19] ve tekstil malzemelerinde [20-24] alev geciktirici olarak kullanılmaktadırlar. Alev geciktiricilerin kullanımı gün geçtikçe artmaktadır dolayısıyla yeni alev geciktiriciler geliştirmek önemli bir araştırma konusu haline gelmiştir. Alev geciktiriciler kimyasal veya fiziksel mekanizmalar yoluyla buhar fazında veya yoğun fazda hareket etmek suretiyle ısıtma, piroliz, ateşleme veya alev yayılması sırasında yanmaya müdahale eder. Borlu bileşikler, $\mathrm{CO}$ veya $\mathrm{CO}_{2}$ oluşumu yerine karbon ayrışma sürecini lehine yeniden yönlendirerek yoğun fazda hareket ederler. Borlu bileşikler, karbon oksidasyonunu önleyen koruyucu camsı koruma tabakasının oluşmasını sağlarlar ve yanan malzemenin üzerini oksijenle temasını kesecek şekilde kaplayarak yanmayı bastırırlar [25]. Bor içeren alev geciktiriciler halojen içeren geleneksel alev geciktiricilere nazaran daha az toksiktirler [26]. Bor bileşikleri alev geciktirici olarak kullanıldıklarında çevre dostu olarak kabul edilirler $[27,28]$. Ayrıca duman bastırmada, yanmayı önlemede ve çok işlevli alev geciktiricilerde boya pigmenti olarak kullanıırlar [29]. Malzemelerin alev geciktiriciliğini analiz eden test metodlarından bazıları LOI analizi, UL-94 testi, termogravimetrik analiz, koni kalorimetre testidir [30].

Bu çalışmanın amacı, fonksiyonel ve yüksek katma değerli bor uç ürünlerinden biri olan kalsiyum floroboratın üretim parametrelerinin belirlenmesi, kinetik ve alev geciktirici özelliklerinin incelenmesidir. Literatürde kalsiyum floroboratın sentez parametrelerinin belirlendiği ve reaksiyon kinetiğinin çalışıldığı ve ayrıca kalsiyum floroboratın pamuklu kumaşta alev geciktirici etkisinin incelendiği bir çalışma bulunmamaktadır.

\section{Malzemeler ve Yöntemler (Materials and Methods)}

Yapılan çalışmada yaş metot yöntemi ile kalsiyum oksit (\%99 saflıkta Sigma-Aldrich) ve floroborik asit (\%50 saflıkta ACROS Organics) reaktant olarak kullanılarak kalsiyum floroborat sentezlenmiştir. Reaksiyon Eş. 1 'de verilmiştir.

$$
\mathrm{CaO}+2 \mathrm{HBF}_{4} \rightarrow \mathrm{Ca}\left(\mathrm{BF}_{4}\right)_{2}+\mathrm{H}_{2} \mathrm{O}
$$

Deneyler, floroborik asitin camlarda korozif etki göstermesinden dolayı teflon reaktörler içerisinde gerçekleştirilmiştir. Çalışılan parametreler; reaktant mol oranı $(2: 1,3: 1,4: 1,5: 1)$, sıcaklık $\left(30^{\circ} \mathrm{C}, 50^{\circ} \mathrm{C}, 70^{\circ} \mathrm{C}, 90^{\circ} \mathrm{C}\right.$, $100^{\circ} \mathrm{C}$ ) ve reaksiyon süresidir. Deneylerde karıştırma hızı 400 rpm (rotation per minute) olarak sabit tutulmuştur. Eş. 1'de verilen reaksiyon denklemine göre oluşabilecek maksimum kalsiyum floroborat miktarı hesaplanıp üretilen madde miktarından verime geçilmiştir. Sentezlenen kalsiyum floroboratın karakterizasyon çalışmalarında FT-IR (Jasco FT-IR-480), XRD (Bruker D8 Advance) ve $\mathrm{BF}_{4}$ - iyon seçici elektrot (Mettler Toledo DX287) kullanılmıştır. Numunenin termal davranışı, TG-DTA (NETZSCH5) cihazı ile argon atmosferinde $10^{\circ} \mathrm{C} /$ dakika hızla oda sıcaklığından $800^{\circ} \mathrm{C}$ 'ye kadar gözlemlenmiştir. Kinetik çalışmada reaksiyonun mertebesi belirlenmiş ve aktivasyon enerjisi hesaplanmıştır. Kalsiyum floroboratın alev geciktirici özelliğinin incelenmesinde limit oksijen indeksi testi (LOI) kullanılmış ve testler ASTM D2863 standardına göre yapılmıştır. Optimum koşullarda sentezlenen kalsiyum floroborat farklı derişimlerde (\%20 ve $\% 50)$ hazırlanarak pamuklu kumaşlara emdirilmiş ve kumaşlar oda sıcaklığında kurutulmuştur. Deneylerde kullanılan kumaşın özellikleri Tablo 1'de verilmiştir.

Tablo 1. LOI testinde kullanılan kumaşın özellikleri (Properties of the fabric used in the LOI test).

\begin{tabular}{ll}
\hline Lif Cinsi & $\% 100$ Pamuk Ring İpliği \\
Dokuma biçimi & $2 \times 2$ \\
Alanın yoğunluğu & $437 \mathrm{~g} / \mathrm{m}^{2}$ \\
İp sıklığı & Atkı yönünde $15 \mathrm{adet} / \mathrm{cm}$ \\
& Çözgü yönünde $23 \mathrm{adet} / \mathrm{cm}$ \\
\hline
\end{tabular}




\section{Sonuçlar ve Tartışma (Results and Discussion)}

Yapılan deneysel çalışmanın ilk aşamasında farklı parametrelerin reaksiyon verimi üzerine etkisi incelenmiştir. Eş.1'de verilen reaksiyon denklemine göre reaktant mol oranının $\left(\mathrm{nHBF}_{4} / \mathrm{nCaO}\right)$ 2:1, 3:1, 4:1, 5:1 olduğu durumlarda reaksiyon verim değerleri hesaplanmıştır. Sabit sıcaklık ve karıştırma hızında $\left(50^{\circ} \mathrm{C}\right.$ ve 400 rpm) 120 dakika boyunca gerçekleşen reaksiyonlarda elde edilen maddeler süzülüp kurutulmuştur. Reaktant mol oranının verim üzerine olan etkisi Şekil 1 'de verilmiştir. Şekil 1'de görüldüğü üzere kalsiyum floroboratın en uygun şartlarda \%74 verimle ile 4:1 reaktant mol oranında elde edildiği gözlemlenmiştir. Reaktant mol oranı arttıkça seyreltme etkisinden kaynaklı verimin azaldığı belirlenmiştir.

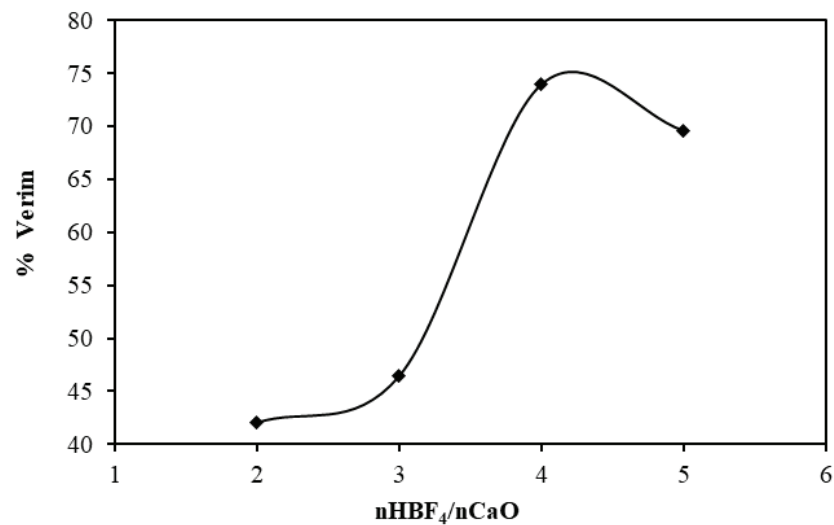

Şekil 1. Reaktant mol oranının $\left(\mathrm{nHBF}_{4} / \mathrm{nCaO}\right)$ reaksiyon verimi üzerine etkisi $\left(50^{\circ} \mathrm{C}\right.$ ve $400 \mathrm{rpm}$ ) (Effect of reactant mole ratio $\left(\mathrm{nHBF}_{4} / \mathrm{nCaO}\right)$ on reaction efficiency $\left(50^{\circ} \mathrm{C}\right.$ ve $\left.\left.400 \mathrm{rpm}\right)\right)$.

Reaksiyonun mol oranı $\left(\mathrm{nHBF}_{4} / \mathrm{nCaO}=4: 1\right)$ ve karıştırma hızı (400 rpm) sabit tutularak farklı sıcaklıklarda $\left(30^{\circ} \mathrm{C}, 50^{\circ} \mathrm{C}, 70^{\circ} \mathrm{C}, 90^{\circ} \mathrm{C}, 100^{\circ} \mathrm{C}\right)$ deneylere devam edilmiş, sıcaklığın reaksiyon verimi üzerine etkisi belirlenmiştir. Farklı sıcaklık değerlerinde elde edilen verim değerleri Şekil 2'de verilmiştir. Şekil 2'de görüldüğü gibi, sıcaklık arttıkça reaksiyon veriminin arttığı gözlemlenmiştir. $90^{\circ} \mathrm{C}$ ve $100^{\circ} \mathrm{C}$ 'de elde edilen verim değerlerinin yaklaşık aynı olduğu görülmüştür. Enerji

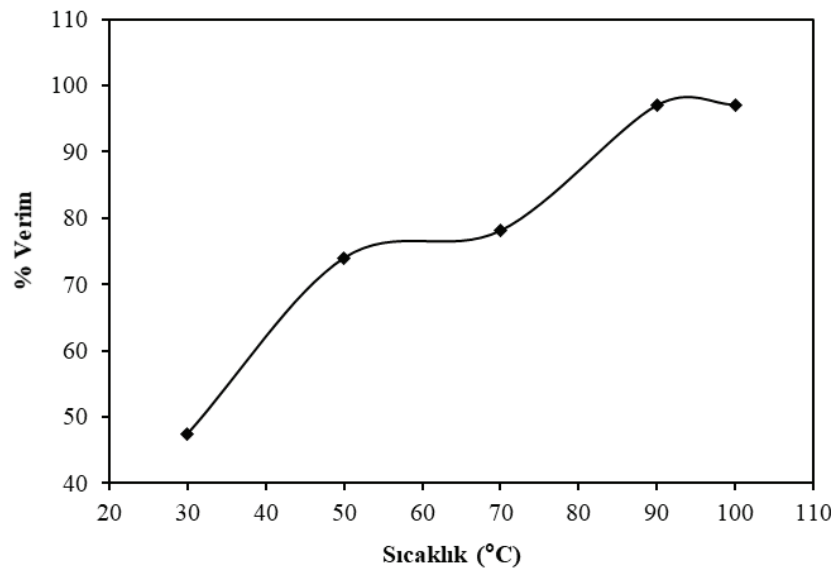

Şekil 2. Sıcaklığın reaksiyon verimi üzerine etkisi $\left(\mathrm{nHBF}_{4} /\right.$ $\mathrm{nCaO}=4: 1,400 \mathrm{rpm}$ ) (Effect of temperature on reaction efficiency $\left(\mathrm{nHBF}_{4} / \mathrm{nCaO}=4: 1,400 \mathrm{rpm}\right)$ ). maliyetleri göz önünde bulundurulduğunda reaksiyon için en uygun sıcaklık $90^{\circ} \mathrm{C}$ olarak belirlenmiştir. $4: 1$ reaktant mol oranında ve $90^{\circ} \mathrm{C}$ 'de kalsiyum floroborat $\% 97$ verim ile sentezlenmiştir.

Çalışmanın bir sonraki basamağında reaksiyon süresinin incelenmesi maksadı ile 4:1 reaktant mol oranı ve $90^{\circ} \mathrm{C}$ sabit sıcaklık şartlarında reaksiyon ortamından 1'er mL numune alınarak 100 mL'ye seyreltilmiştir. $\mathrm{BF}_{4}$ - iyon seçici elektrot kullanılarak $\mathrm{BF}_{4}{ }^{-}$konsantrasyonu iyon metrede okunmuştur. $\mathrm{BF}_{4}-$ konsantrasyonunun zamana bağlı değişimi Şekil 3'te verilmiştir. $\mathrm{BF}_{4}$ - konsantrasyonunun zamanla arttığı fakat 100 . dakikadan sonra yaklaşık aynı değerde kaldığı gözlemlenmiştir. Dolayısı ile reaksiyon süresi 100 dakika olarak belirlenmiştir.

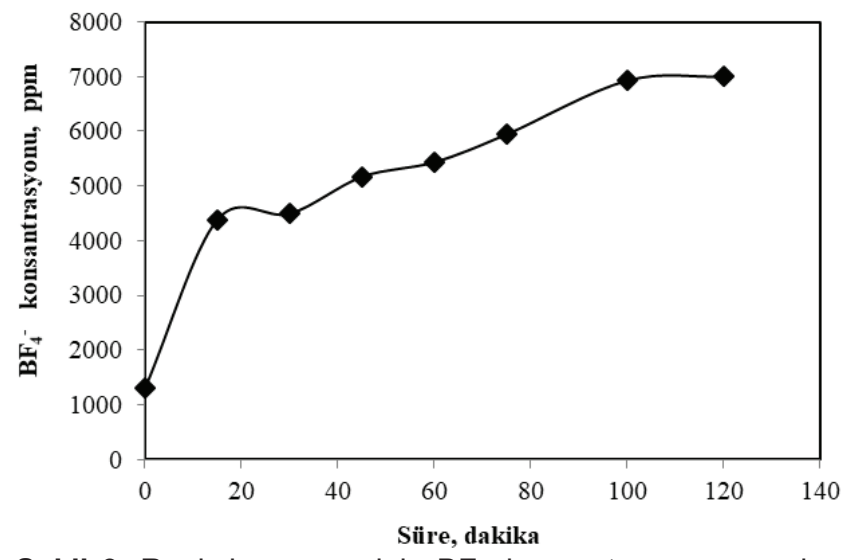

Şekil 3. Reaksiyon süresinin $\mathrm{BF}_{4}$ - konsantrasyonu üzerine etkisi $\left(\mathrm{nHBF}_{4} / \mathrm{nCaO}=4: 1,90^{\circ} \mathrm{C}\right)\left(\right.$ Effect of reaction time on $\mathrm{BF}_{4}$ concentration $\left.\left(\mathrm{nHBF}_{4} / \mathrm{nCaO}=4: 1,90^{\circ} \mathrm{C}\right)\right)$.

Yaş yöntem ile en uygun koşullarda sentezlenen kalsiyum floroboratın saflaştırma işlemi için kalsiyum floroboratın suda çözünürlüğünden yararlanılmıştır. Elde edilen ürün $40^{\circ} \mathrm{C}$ sıcaklıktaki suda çözülmüş, süzülmüş ve kurutulmuştur. Daha sonra sentezlenen kalsiyum floroborattan $0,03 \mathrm{~g}$ alınmış ve $0,47 \mathrm{~g} \mathrm{KBr}$ ile karıştırılarak pellet oluşturulmuş ve FT-IR cihazında analiz edilmiştir. FT-IR spektrumunda B-F bağı karakteristik piki $1000-1100 \mathrm{~cm}^{-1}$ aralığında görülmektedir [31]. Sentezlenen numunenin FT-IR spektrumu Şekil 4'te verilmiştir. Şekil 4'te verilen FT-IR spektrumunda literatür verisiyle örtüşecek şekilde $1000-1100 \mathrm{~cm}^{-1}$ aralığında B-F bağı piki görülmektedir. $541 \mathrm{~cm}^{-1}$ dalga

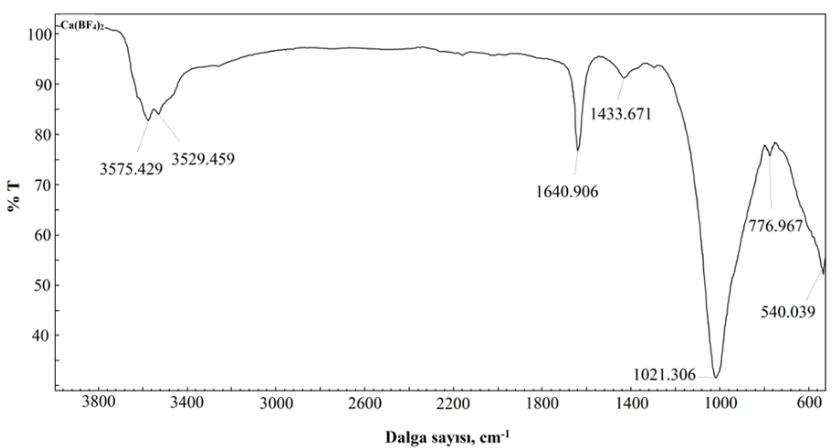

Şekil 4. Sentezlenen kalsiyum floroboratın FTIR spektrumu (FTIR spectrum of synthesized calcium fluoroborate). 
sayısında B-F geriliminden kaynaklanan titreşim mevcuttur. $3500-3600 \mathrm{~cm}^{-1}$ dalga sayısı aralığında ise $\mathrm{O}-\mathrm{H}$ pikleri belirmiştir.

Şekil 5'te sentezlenen kalsiyum floroboratın kristal yapısını gösteren XRD analizi grafiği verilmiştir (ICSD/98-000-1839). 12,6 $13,2^{\circ}$ ve $19,1^{\circ}$ değerlerinde gözlemlenen piklerin literatür verileriyle örtüştüğü görülmektedir [11]. Bu durum reaksiyonun başarılı bir şekilde gerçekleştiğini göstermektedir.

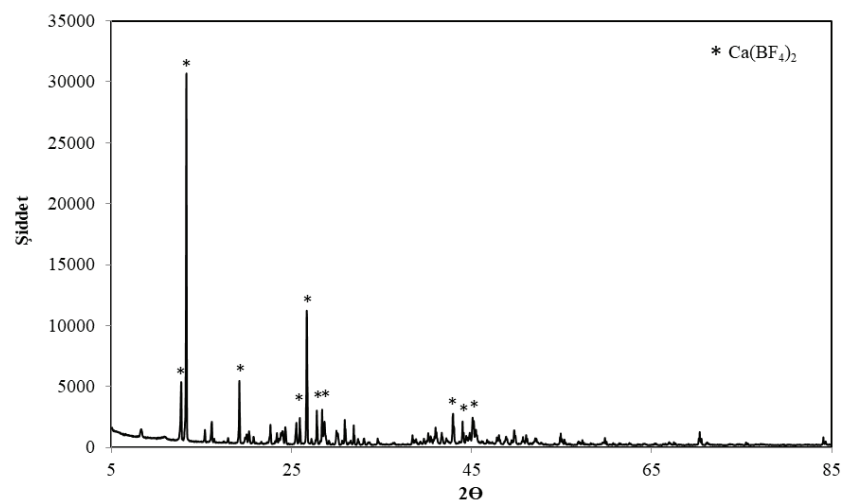

Şekil 5. Sentezlenen kalsiyum floroboratın XRD analiz grafiği (XRD analysis graph of synthesized calcium fluoroborate).

Sentezlenen kalsiyum floroboratın TG-DTA grafiği Şekil 6'da verilmiştir. İlk olarak yapıdan su buharlaşmıştır. $300^{\circ} \mathrm{C}$ 'de bağların parçalanması ve gaz çıkışından dolayı yapıda \%61 kütle kaybı olmuştur. Yüksek sıcaklıklarda borlu yapı, camsı tabaka oluşturmaktadır. İnorganik bor bileşikleri yanma esnasında malzeme yüzeyinde camsı koruyucu tabaka oluşturarak, yanma için gerekli oksijen ve ısıya karşı koruma görevi yapmaktadırlar [32].

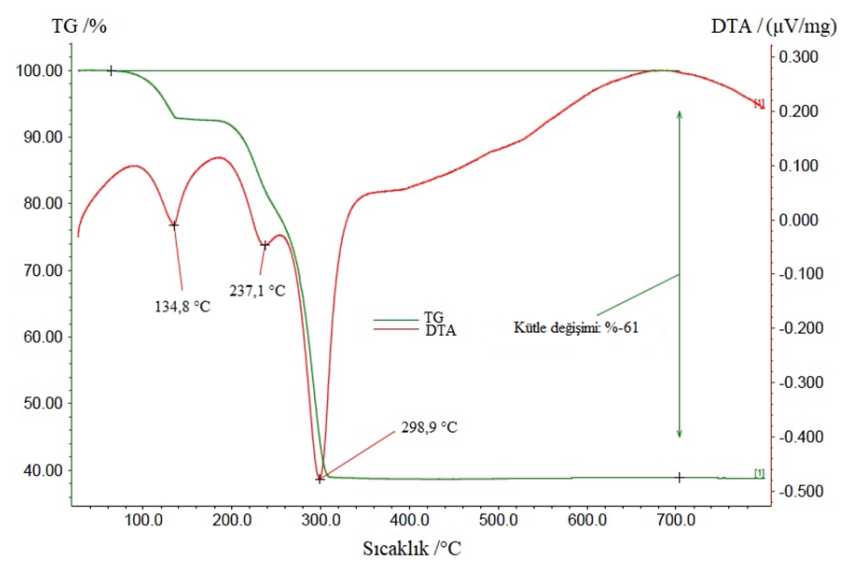

Şekil 6. Sentezlenen kalsiyum floroboratın TG-DTA grafiği (TG-DTA graph of synthesized calcium fluoroborate).

Yapılan kinetik çalışmada reaksiyonun mertebesini belirlemek için her bir sıcaklıkta $\ln \left(\mathrm{C}_{\mathrm{A}} / \mathrm{C}_{\mathrm{A} 0}\right)$ 'a karşı $\mathrm{T}$ grafiği çizilmiş ve reaksiyon mertebesinin birinci dereceden olduğu bulunmuştur. $\ln \left(\mathrm{C}_{\mathrm{A}} / \mathrm{C}_{\mathrm{A} 0}\right)$ 'a karşı T grafiklerinin eğimlerinden $\mathrm{k}$ değerleri bulunmuştur. Aktivasyon enerjisinin belirlenmesinde beş farklı sıcaklık için Ink' ya karşı 1/T değerleri grafiğe geçirilerek doğrusal bir çizgi elde edilmiştir. Kalsiyum floroboratın Ink'ya karşı 1/T Arrhenius grafiği Şekil 7'de verilmiştir. Bu doğrunun eğiminden $-E_{a} / R$ elde edilmiş ve aktivasyon enerjisi $\left(E_{a}\right)$ değeri bulunmuştur.

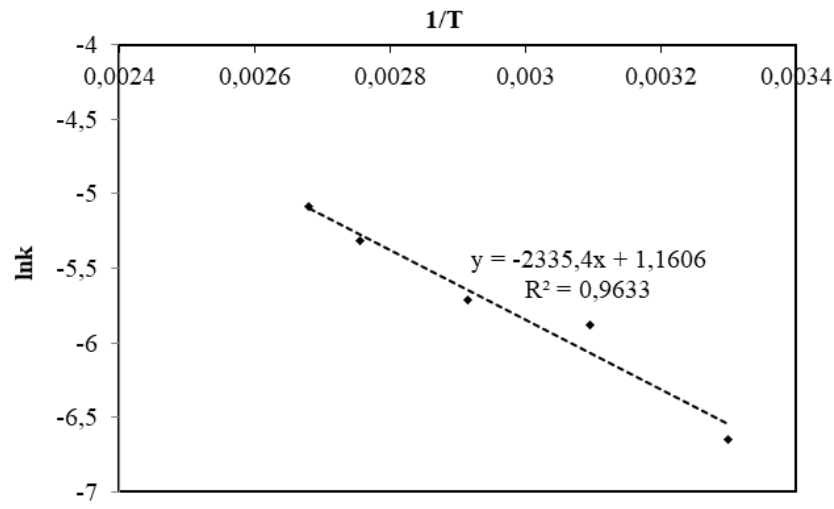

Şekil 7. Kalsiyum floroborat Arrhenius grafiği (Arrhenius plot of calcium fluoroborate).

Yapılan kinetik çalışma sonucunda reaksiyonun aktivasyon enerjisi $19,14 \mathrm{~kJ} / \mathrm{mol}$ olarak bulunmuştur. Bu sonuç elde edilen kalsiyum floroboratın üretim sürecinin enerji tüketimi bakımından ekonomik olduğunu göstermektedir. Ayrıca üretim aşamasında yüksek sıcaklığa intiyaç yoktur. Zira düşük aktivasyon enerjisine sahip süreçler sıcaklığa çok duyarlı değildir. Kinetik çalışma ve diğer sonuçlar birlikte değerlendirildiğinde reaksiyonun teknolojik olarak uygulanabilir olduğu anlaşılmaktadır.

Kalsiyum floroboratın alev geciktirici özelliğini incelemek için sentezlenen kalsiyum floroborat kristallerinin farklı derişimlerde (\%20 ve \%50) çözeltileri hazırlanmış ve \%100 pamuklu kumaşlara emdirilmiştir. Ardından katkısız kumaşın ve kalsiyum floroborat çözeltisi emdirilmiş kumaşların LOI değerleri belirlenmiştir. Bir malzemeyi alev geciktirici olarak nitelendirilebilmek için, limit oksijen indeks değerinin \%28'in üzerinde olması gerektiği belirtilmiştir [33]. Limit oksijen indeksi, malzemenin havada yanmaya devam etmesi için gerekli olan \% oksijen miktarı olarak tanımlanabilmektedir. LOI değeri yüksek olan malzeme standart atmosfer şartları altında daha zor yanma karakteristiğine sahiptir.

ASTM D 2863 standardına göre yapılan testlerde fazla güçlü olmayan sabit miktardaki alev, malzemenin uç kısmına gerekirse üst yüzeyini kaplayacak şekilde sürekli hareket ettirilmek sureti ile 30 saniye kadar uygulanmış ve her 5 saniyede bir uzaklaştırılarak numunenin kendiliğinden yanmaya devam edip etmeyeceği gözlemlenmiştir. LOI testi sonucunda elde edilen sonuçlar Tablo 2'de verilmiştir. Tablo 2'de görüldüğü üzere katkısız pamuklu kumaşının LOI değeri 16 iken, \%20'lik kalsiyum floroborat çözeltisi emdirilen kumaşın LOI değeri 23, \%50'lik kalsiyum floroborat çözeltisi emdirilen kumaşın LOI değeri ise 32'dir. Literatürde \%50 derişimdeki amonyum floroborat çözeltisinin katkısız kumaşın LOI değerini \%22 arttırdığı görülmüştür [24]. \%20 derişimdeki bakır floroborat çözeltisinin katkısız 
Tablo 2. Farklı derişimlerde kalsiyum floroborat çözeltisi emdirilen kumaşların LOI değerleri (LOI values of fabrics impregnated with calcium fluoroborate solution at different concentrations).

\begin{tabular}{|c|c|c|c|}
\hline Numune Adı & Katkısız Kumaş & $\begin{array}{c}\text { \%20'lik } \\
\mathrm{Ca}\left(\mathrm{BF}_{4}\right)_{2} \text { Çözeltisi Emdirilmiş } \\
\text { Kumaş }\end{array}$ & $\begin{array}{c}\text { \%50'lik } \\
\mathrm{Ca}\left(\mathrm{BF}_{4}\right)_{2} \text { Çözeltisi Emdirilmiş } \\
\text { Kumaş }\end{array}$ \\
\hline LOI değeri & 16 & 23 & 32 \\
\hline
\end{tabular}

kumaşın LOI değerini \%75 arttırdığı, \%30 derişimdeki kobalt floroborat çözeltisinin ise katkısız kumaşın LOI değerini iki katından fazla arttırdığı görülmüştür $[34,35]$. Kalsiyum floroboratın pamuklu kumaşın LOI değerini yani alevin devam edebilmesi için gerekli olan oksijen miktarını iki katı kadar yükselttiği ve kumaşa alev geciktirici özellik kazandırdığı görülmüştür.

\section{Sonuçlar (Conclusions)}

Yapılan çalışmada kalsiyum oksit ile floroborik asit reaktant olarak kullanılarak kalsiyum floroborat yaş yöntem ile sentezlenmiştir. Reaktant mol oranı, sıcaklık ve reaksiyon süresinin verim üzerine etkisi incelenmiştir. 4:1 reaktant mol oranı, $90^{\circ} \mathrm{C}$ sıcaklık ve 100 dakika reaksiyon süresinde $\% 97$ verimle kalsiyum floroborat sentezlenmiştir. Kinetik çalışmada reaksiyon derecesinin birinci dereceden kinetiğe sahip olduğu belirlenmiş ve aktivasyon enerjisinin $\left(E_{a}\right) 19,14 \mathrm{~kJ} / \mathrm{mol}$ olarak hesaplanmıştır. Bu sonuçlar reaksiyonun ekonomik olduğunu göstermektedir. Teknolojik açıdan uygulanabilirliği ve fizibilitesi yüksektir. LOI testi maddelerin alev geciktirici karakteristiğini ortaya koyan önemli testlerden biridir. Kalsiyum floroborat emdirilmiş kumaşlara LOI testi uygulanmış, alev geciktirici özellikleri incelenmiştir. Katkısız kumaşın LOI değeri 16 bulunurken; $\% 20$ ve \%50 kalsiyum floroborat içeren çözeltiler emdirilip kurutulmuş kumaşların LOI değerleri sırasıyla 23 ve 32 olarak bulunmuştur. LOI testi sonuçları kalsiyum floroboratın alev geciktirici özelliğe sahip olduğunu göstermektedir. Yüksek katma değerli özel bor ürünlerinden olan kalsiyum floroboratın tekstil kumaşlarında alev geciktirici olarak kullanımının yaygınlaştırılması önerilmektedir

\section{Teşekkür (Acknowledgement)}

Bu çalışma, TENMAK Bor Araştırma Enstitüsü (BOREN) tarafından desteklenmiştir. Proje No: 2017-3006-30-002.

\section{Kaynaklar (References)}

[1] Türk Mühendisler Mimarlar Odaları Birliği. (2016). Bor Raporu. [Boron Report]. ISBN 978-605-01-0883-5.

[2] Booth, H. S., \& Martin, D. R. (1949). Boron Trifluoride and Its Derivatives. Wiley, Newyork. DOI: 10.1021/ ed028p56.

[3] Yünlü, K. (2019). Bor: Bileşikleri, Sentez Yöntemleri, Özellikleri, Uygulamaları. [Boron: Its Compounds, Synthesis Methods, Properties and Applications] (2nd Ed.). Aydili Advertising Agency. ISBN 978-605-531093-6.
[4] Achilonu, M. C., \& Umesiobi, D. O. (2010). The formation of carbon-carbon and carbon-heteroatom bonds using silver tetrafluoroborate as a promoter. Arabian Journal of Chemistry, 9(2), 1984-2003.

[5] Aydın, Y. D., Gürü, M., İpek, D., \& Özyürek, D. (2019). Obtainment of copper(II) fluoroborate by high-energy impacted ball-milling. Acta Physica Polonica A, 135, 888-891.

[6] Aydın, D. Y., Gürü, M., Ipek, D., \& Özyürek, D. (2017). Synthesis and characterization of zinc fluoroborate from zinc fluoride and boron by mechanochemical reaction. Arabian Journal for Science and Engineering, 42(10), 4409-4416.

[7] Krishnapuram, P., Jakka, S. K., Thummala, C., \& Lalapeta, R. M. (2012). Photoluminescence characteristics of Eu2O3 doped calcium fluoroborate glasses. Journal of Molecular Structure, 1028, 170-175.

[8] Kumar, J. S., Pavani, K., Babu, A. M., Giri, N. K., Rai, S.B., \& Moorthy, L. R. (2010). Fluorescence characteristics of Dy3+ ions in calcium fluoroborate glasses. Journal of Luminescence, 130 (10), 1916-1923.

[9] Kumar, J. S., Babu, A. M., Sasikala, T., Moorthy, \& L. R. (2010). NIR fluorescence and visible upconversion studies of $\mathrm{Nd} 3+$ ions in calcium fluoroborate glasses. Chemical Physics Letters, 484(4-6), 207-213.

[10] Prabakar, S. J. R., Sohn, K.S., \& Pyo, M. (2019). Enhancement in high-rate performance of graphite anodes via interface modification utilizing $\mathrm{Ca}(\mathrm{BF} 4) 2$ as an electrolyte additive in lithium ion batteries. Journal of The Electrochemical Society, 166, A591.

[11] Forero-Saboya, J. D., Lozinšek, M., \& Ponrouch, A. (2020). Towards dry and contaminant free $\mathrm{Ca}(\mathrm{BF} 4) 2-$ based electrolytes for $\mathrm{Ca}$ plating. Journal of Power Sources Advances, 6, 100032.

[12] Sarkar, A., Santra, S., Kundu, S. K., Ghosal, N. C. Hajra, A., \& Majee, A. (2015). Zinc tetrafluoroborate: a versatile and robust catalyst for various organic reactions and transformations. Synthesis, 47,1379-1386.

[13] Pujala, B., Rana, S., \& Chakraborti, A. K. (2011). Zinc tetrafluoroborate hydrate as a mild catalyst for epoxide ring opening with amines: scope and limitations of metal tetrafluoroborates and applications in the synthesis of antihypertensive drugs (RS)/(R)/(S)-metoprolols. The Journal of Organic Chemistry, 76(21), 8768-8780.

[14] Ranu, B. C., Jana, U., \& Majee, A. (1999). A simple and efficient method for selective deprotection of $t$-butyldimethylsilyl ethers by zinc tetrafluoroborate in water. Tetrahedron Letters, 40(10), 1985-1988.

[15] Zhang, S. S., Xu, K., \& Jow, T. R. (2002). Study of LiBF4 as an electrolyte salt for a li-ion battery. Journal of The Electrochemical Society, 149(5), A586-A590.

[16] Tressaud, A. (2010). Functionalized Inorganic Fluorides: Synthesis, Characterization and Properties of Nanostructured Solids. John Wiley \& Sons. ISBN 9780-470-74050-7. 
[17] Hashmi S. (Ed.) (2014). Comprehensive Materials Processing, Elsevier. ISBN 978-0-08-096533-8

[18] Menz, W., Mohr, J., \& Paul, O. (2008). Microsystem Technology. John Wiley \& Sons. ISBN 9783527296347.

[19] Wilkie, C. A., \& Morgan, A. B. (Eds.). (2009). Fire Retardancy of Polymeric Materials. CRC Press.

[20] Aydın, D. Y. (2015) Çinko floroborat sentezi ve alev geciktirici olarak kullanılabilirliği [Synthesis of zinc fluoroborate and usability as flame retardant]. Yüksek Lisans Tezi, Gazi Üniversitesi, [M. Sc. Thesis, Gazi University]. Council of Higher Education Thesis Center (Thesis Number 395758)..

[21] Aydın, D. Y., Patlar, K., Gürü, \& M., Akkurt, F. (2017). Synthesis of antimony fluoroborate and usability as flame retardant, 8th International Advanced Technologies Symposium (IATS'17), Elazığ, Turkey.

[22] Aydın, D. Y., Kurt, H., Kandemir, E. B., \& Gürü, M. (2017) Synthesis of lead fluoroborate and usability as flame retardant, 8th International Advanced Technologies Symposium (IATS'17), Elazığ, Turkey.

[23] Aydın, D. Y., Biberoğlu, R., Kümbetlioğlu, F., Gürü, M., Akkurt F., \& Kuru, F. (2018) Kobalt floroborat sentezi ve alev geciktirici olarak kullanılabilirliği [Synthesis of cobalt fluoroborate and its usability as a flame retardant] 1st International Eurasion Conference on Science, Engineering and Technology, Ankara, Turkey.

[24] Ceyhan, A. A., Bağcı, S., Baytar, O., \& Şahin, Ö. (2020). Amonyum floroborat üretimi ve üretim parametrelerinin belirlenmesi [Ammonium fluoroborate production and determination of production parameters]. Journal of Boron, 5(2), 63-72.

[25] Aydın, D. Y., Gürü, M., Ayar, B., \& Çakanyıldırım, Ç. (2016). Bor bileşiklerinin alev geciktirici ve yüksek sıcaklığa dayanıklı pigment olarak uygulanabilirliği. [Applicability of boron compounds as flame retardant and high temperature resistant pigments]. Journal of Boron, 1(1), 33-39.

[26] Lu, S. Y., \& Hamerton I. (2002) Recent developments in the chemistry of halogen-free flame retardant polymers. Progress in Polymer Science, 27(8), 1661-1712.

[27] Dogan, M., \& Unlu, M. (2014). Flame retardant effect of boron compounds on red phosphorus containing epoxy resins. Polymer Degradation and Stability, 99, 12-17.

[28] Wang, X, Song, Y., \& Bao, J. (2010). Synergistic effects of $\mathrm{ZrO} 2$ or B2O3 on flame-retarded poly (butyl methacrylate) with tricresylphosphate. Fire and Materials, 34(7), 357-366.

[29] Ayar, B., Gürü, M., \& Çakanyıldırım, Ç. (2014). Solid phase synthesis of anhydrous zinc borate from zinc and boron oxide and utilization as a flame retardant in dye and textile. Gazi University Journal of Science, 27(3), 987-991.

[30] Bourbigot, S., Bras, M. L., Leeuwendal, R., Shenc, K. K., \& Schubertc, D. (1999). Recent advances in the use of zinc borates in flame retardancy of EVA. Polymer Degradation and Stability, 64(3), 419-425.

[31] Leoni, P., Sommovigo, M., Pasqualli, M., Midollini, S., Braga, D., \& Sabatino, P. (1991). Coordinated water/ anion hydrogen bonds and $\mathrm{Pd}-\mathrm{H}$ bond acidity in cationic palladium(II) aquo hydrides and the x-ray crystal and molecular structures of trans-[(Cy3P $) 2 \mathrm{Pd}(\mathrm{H})$
$(\mathrm{H} 2 \mathrm{O})] \mathrm{BF} 4(\mathrm{Cy}=$ cyclohexyl). Organometallics, 10(4), 1038-1044.

[32] Bozacı, E. (2018). Borlu bileşiklerin çevre dostu yöntemlerle poliakrilnitril kumaşlara uygulanması [Application of boron compounds to polyacrylonitrile fabrics by environmentally friendly methods]. Journal of Boron, 3(1), 17-23.

[33] Friedrich, K., Breuer, U. (2015) Multifunctionality of Polymer Composites: Challenges and New Solutions. (1st Ed.), Elsevier, ISBN 978-0-323-26434-1.

[34] İpek, D., (2018). Bakır floroborat sentezi ve alev geciktirici olarak kullanılabilirliği [Synthesis of copper fluoroborate and usability as flame retardant]. Yüksek Lisans Tezi, Gazi Üniversitesi, [M. Sc. Thesis, Gazi University]. Council of Higher Education Thesis Center (Thesis Number 493623).

[35] Biberoğlu, R. (2020). Kobalt floroborat sentezi ve alev geciktirici olarak kullanılabilirliği [Synthesis of cobalt fluoroborate and usability as flame retardant]. [M. Sc. Thesis, Gazi University]. Council of Higher Education Thesis Center (Thesis Number 634601). 